\title{
Quantum lattice fluctuations in a frustrated Heisenberg spin-Peierls chain*
}

\author{
A. Weiße ${ }^{a}$, G. Wellein ${ }^{b}$ and H. Fehske ${ }^{c}$ \\ ${ }^{a}$ Physikalisches Institut, Universität Bayreuth, 95440 Bayreuth, Germany \\ ${ }^{b}$ Regionales Rechenzentrum Erlangen, Universität Erlangen, 91058 Erlangen, Germany \\ ${ }^{c}$ Theoretische Physik, Universität des Saarlandes, 66041 Saarbrücken, Germany
}

(January 16, 2014)

\begin{abstract}
As a simple model for spin-Peierls systems we study a frustrated Heisenberg chain coupled to optical phonons. In view of the anorganic spin-Peierls compound $\mathrm{CuGeO}_{3}$ we consider two different mechanisms of spin-phonon coupling. Combining variational concepts in the adiabatic regime and perturbation theory in the anti-adiabatic regime we derive effective spin Hamiltonians which cover the dynamical effect of phonons in an approximate way. Ground-state phase diagrams of these models are determined, and the effect of frustration is discussed. Comparing the properties of the ground state and of low-lying excitations with exact diagonalization data for the full quantum spin phonon models, good agreement is found especially in the anti-adiabatic regime.
\end{abstract}

PACS numbers: 75.10.Jm, 63.20.Kr

\section{INTRODUCTION}

The effect of a Peierls instability in quasi-onedimensional spin systems, i.e. the instability of a uniform spin chain towards dimerization induced by the interaction with lattice degrees of freedom, has attracted considerable attention over the last decades. Starting in the seventies with organic compounds of the TTF and TCNQ family [1], the interest in the Peierls instability was renewed with the discovery of a spin-Peierls (SP) transition in the anorganic compound $\mathrm{CuGeO}_{3}$ in 1993 by Hase et al. [2]. The most significant feature distinguishing $\mathrm{CuGeO}_{3}$ from other SP-compounds is the high energy of the involved optical phonons, which is comparable to the magnetic exchange integral $J$. In contrast to the organic materials no softening of these phonon modes is observed near the transition. Therefore the adiabatic treatment of the phonon subsystem used in the works of Pytte [3] or Cross and Fisher [4], does not seem appropriate to describe the SP transition in $\mathrm{CuGeO}_{3}$, although there are recent efforts in this direction [5]. Rather one has to take into account the effect of quantum lattice fluctuations which tend to decrease the SP transition temperature respectively the energy gap between the ground state and lowest excitations in the dimerized phase. Unfortunately there are practically no analytic methods to handle coupled systems of spins (electrons) and phonons when all energy scales and coupling strengths are of the same order of magnitude. This is why many studies involving dynamical phonons rely on numerical methods, such as exact diagonalization (ED) [6,7], density matrix renormalization group (DMRG) $[8,9]$ or Monte Carlo simulation (MC) [10]. Only recently Zheng [11] developed an analytical approach to describe the SP instability of a XY

\footnotetext{
*Dedicated to H. Büttner on the occasion of his 60 th birthday.
}

spin chain, which is based on the unitary transformation method. It works well in the adiabatic and in the antiadiabatic regime. In the latter case there are also some approaches to the Heisenberg spin chain interacting with optical phonons: Kuboki and Fukuyama [12] used perturbation theory to derive an effective spin Hamiltonian, while Uhrig [13] applied the flow-equation method [14].

As a simple model which contains all important features of a SP system in the following we consider an antiferromagnetic Heisenberg chain coupled to a set of Einstein oscillators,

$$
H=H_{\mathrm{s}}+H_{\mathrm{p}}+H_{\mathrm{sp}}
$$

with

$$
\begin{aligned}
& H_{\mathrm{s}}=J \sum_{i}\left(\mathbf{S}_{i} \cdot \mathbf{S}_{i+1}+\alpha \mathbf{S}_{i} \cdot \mathbf{S}_{i+2}\right), \\
& H_{\mathrm{p}}=\omega_{0} \sum_{i} b_{i}^{+} b_{i} .
\end{aligned}
$$

The interaction of spins and phonons, $H_{\mathrm{sp}}$, can be modelled in two different ways,

$$
\begin{aligned}
& H_{\mathrm{sp}}^{\mathrm{loc}}=\bar{g} \sum_{i}\left(b_{i}^{+}+b_{i}\right) \mathbf{S}_{i} \cdot \mathbf{S}_{i+1}, \\
& H_{\mathrm{sp}}^{\mathrm{diff}}=\bar{g} \sum_{i}\left(b_{i}^{+}+b_{i}\right)\left(\mathbf{S}_{i} \cdot \mathbf{S}_{i+1}-\mathbf{S}_{i} \cdot \mathbf{S}_{i-1}\right),
\end{aligned}
$$

where $\mathbf{S}_{i}$ denote spin- $\frac{1}{2}$ operators at lattice site $i$, while $b_{i}^{+}$and $b_{i}$ are phonon creation and annihilation operators, respectively. $H_{\mathrm{sp}}^{\text {loc }}$ and $H_{\mathrm{sp}}^{\text {diff }}$ differ in the mechanism, how the lattice influences the exchange integral. For $H_{\mathrm{sp}}^{\text {loc }}$, the local coupling, one can think of a single harmonic degree of freedom directly modifying the magnetic interaction. In the context of $\mathrm{CuGeO}_{3}$ this could correspond to side group effects (by the Germanium atoms) as discussed in Ref. [15]. In the case of $H_{\mathrm{sp}}^{\text {diff }}$, the difference coupling, the exchange depends directly on the spatial distance between neighbouring spins. Note that it is not possible to 
uniformly decrease or increase all exchange integrals with this type of spin phonon interaction.

Although $H_{\mathrm{sp}}^{\text {loc }}$ seems to be more appropriate for $\mathrm{CuGeO}_{3}$ we will consider both variants and compare their properties. In addition we take into account a frustrating next-nearest neighbour interaction $J \alpha$, which in view of $\mathrm{CuGeO}_{3}$ was introduced to explain susceptibility data [29]. As we will see below, the spin-phonon interaction is able to induce this kind of long ranged exchange as well.

Motivated by the success of methods combining unitary transformations with variational and numerical techniques, which we used to study the Peierls transition in the Holstein model of spinless fermions [16], and inspired by the work of Zheng [11], in this article we analyse the model (1) within the same framework. In particular we focus on the ground-state phase diagram as a function of spin-phonon coupling, frustration and phonon frequency, and compare our results with exact diagonalization data.

\section{EFFECTIVE SPIN MODELS}

To describe a static lattice dimerization in the adiabatic case of small phonon frequency $\omega_{0}$ we start with a unitary transformation of $H$ which shifts the equilibrium position of each oscillator by a constant amount alternating from site to site, $\tilde{H}=\exp \left(S_{1}\right) H \exp \left(-S_{1}\right)$ with

$$
S_{1}=\frac{\Delta_{\pi}}{2 \bar{g}} \sum_{i}(-1)^{i}\left(b_{i}^{+}-b_{i}\right) .
$$

For the terms involving phonons this yields

$$
\begin{aligned}
\tilde{H}_{\mathrm{p}}= & H_{\mathrm{p}}-\omega_{0} \frac{\Delta_{\pi}}{2 \bar{g}} \sum_{i}(-1)^{i}\left(b_{i}^{+}+b_{i}\right) \\
& +N \omega_{0}\left(\frac{\Delta_{\pi}}{2 \bar{g}}\right)^{2}, \\
\tilde{H}_{\mathrm{sp}}^{\mathrm{loc}}= & H_{\mathrm{sp}}^{\mathrm{loc}}-\Delta_{\pi} \sum_{i}(-1)^{i} \mathbf{S}_{i} \cdot \mathbf{S}_{i+1}, \\
\tilde{H}_{\mathrm{sp}}^{\mathrm{diff}}= & H_{\mathrm{sp}}^{\mathrm{diff}}-2 \Delta_{\pi} \sum_{i}(-1)^{i} \mathbf{S}_{i} \cdot \mathbf{S}_{i+1} .
\end{aligned}
$$

$\Delta_{\pi}$ will act as the variational parameter describing the dimerization of the system.

Next we want to decouple spin and phonon degrees of freedom. The idea is to apply another unitary transformation $\bar{H}=\exp \left(S_{2}\right) \tilde{H} \exp \left(-S_{2}\right)$, where $S_{2}$ should be chosen such that all contributions of first order in the coupling constant $\bar{g}$ disappear in the transformed Hamiltonian. This gives the usual condition for a SchriefferWolff transformation [17],

$$
\tilde{H}_{\mathrm{sp}}+\left.\left[S_{2}, \tilde{H}_{\mathrm{s}}+\tilde{H}_{\mathrm{p}}\right]\right|_{\Delta_{\pi}=0} \stackrel{!}{=} 0
$$

or

$$
S_{2}=\frac{|n\rangle\left\langle n\left|\tilde{H}_{\mathrm{sp}}\right| m\right\rangle\langle m|}{E_{n}-E_{m}},
$$

where $|n\rangle$ and $|m\rangle$ denote eigenstates of the unperturbed Hamiltonian $H_{\mathrm{s}}+H_{\mathrm{p}}$ with the corresponding eigenenergies $E_{n}$ and $E_{m}$. Since we do not know the exact eigenstates of $H_{\mathrm{s}}$, equation (11) can not be written in a simple form. One first guess which describes the phonon-part of $S_{2}$ in a correct way and ensures $S_{2}$ to be antihermitean is

$$
\begin{aligned}
S_{2}^{\text {loc }} & =f \frac{\bar{g}}{\omega_{0}} \sum_{i}\left(b_{i}^{+}-b_{i}\right) \mathbf{S}_{i} \cdot \mathbf{S}_{i+1} \\
S_{2}^{\text {diff }} & =f \frac{\bar{g}}{\omega_{0}} \sum_{i}\left(b_{i}^{+}-b_{i}\right)\left(\mathbf{S}_{i} \cdot \mathbf{S}_{i+1}-\mathbf{S}_{i} \cdot \mathbf{S}_{i-1}\right) .
\end{aligned}
$$

Comparing with (11) $\omega_{0}$ gives the appropriate contribution to the energy denominator, since $H_{\mathrm{sp}}$ connects only phonon states differing by one in their occupation number. What is missing is the contribution of spin states which have overlap via $H_{\mathrm{sp}}$. Therefore, using a numerical procedure we fix the free parameter $f$ by the condition, that the amplitude of the state resulting from the application of $\tilde{H}_{\mathrm{sp}}+\left.\left[S_{2}, \tilde{H}_{\mathrm{s}}+\tilde{H}_{\mathrm{p}}\right]\right|_{\Delta_{\pi}=0}$ to the ground state of $H_{\mathrm{s}}+H_{\mathrm{p}}$ is minimal. Figure 1 illustrates the variation of $f$ with varying phonon frequency $\omega_{0}$. We find that

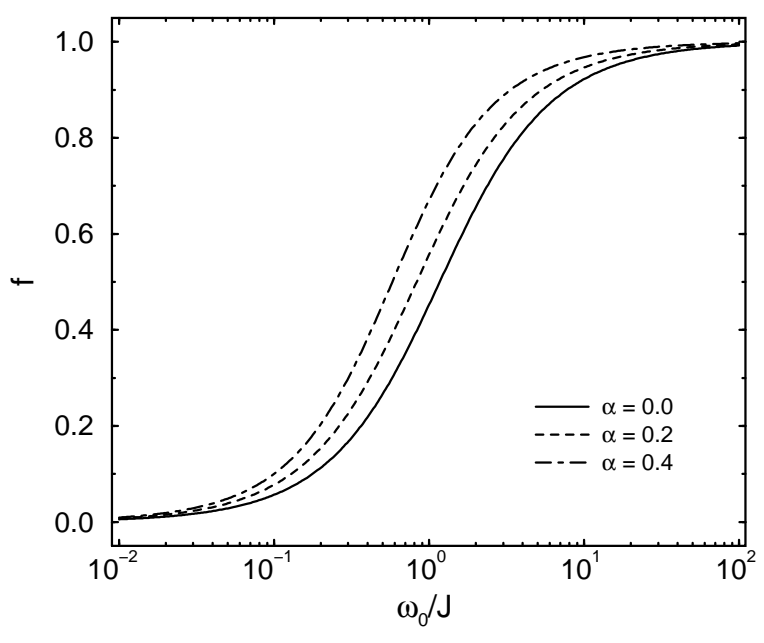

FIG. 1.: Variation of $f$ in the case of local coupling and lattice size $N=16$.

the general shape of $f=f\left(\omega_{0}\right)$ depends only weakly on both, system size $N$ and frustration $\alpha$. While $f \rightarrow 1$ in the anti-adiabatic frequency range $\left(\omega_{0} \gg J\right)$, the transformation $\exp \left(S_{2}\right)$ vanishes completely as the frequency becomes small $\left(\omega_{0} \ll J\right)$.

In contrast to electron-phonon systems with Holstein coupling, where a transformation similar to $\exp \left(S_{2}\right)$ completely removes the electron-phonon interaction term, applying the unitary transformation $\exp \left(S_{2}\right)$ to $\tilde{H}$, we ob- 
tain an infinite series of terms, which can not be summed up to a simple expression, i.e.

$$
\bar{H}=\sum_{k}\left[S_{2}, \tilde{H}\right]_{k} / k !,
$$

where $\left[S_{2}, \tilde{H}\right]_{k}$ denotes the iterated commutator $\left[S_{2}, \tilde{H}\right]_{k+1}=\left[S_{2},\left[S_{2}, \tilde{H}\right]_{k}\right]$ with $\left[S_{2}, \tilde{H}\right]_{0}=\tilde{H}$.

To get an effective spin model we consider only contributions up to fourth order in $\bar{g}$ and average over the phonon subsystem. The resulting spin Hamiltonian $H_{\text {eff }}=\langle\bar{H}\rangle$ contains long ranged Heisenberg interactions as well as numerous four- and six-spin couplings of the form $\left(\mathbf{S}_{i} \cdot \mathbf{S}_{j}\right)\left(\mathbf{S}_{k} \cdot \mathbf{S}_{l}\right) \ldots\left(\mathbf{S}_{m} \cdot \mathbf{S}_{n}\right)$. To a good approximation we can neglect them and obtain

$$
\begin{aligned}
H_{\mathrm{eff}} / J= & J_{0}+\sum_{i}\left(J_{1}+(-1)^{i} \delta\right) \mathbf{S}_{i} \cdot \mathbf{S}_{i+1} \\
& +\sum_{i} \sum_{k=2}^{4} J_{k} \mathbf{S}_{i} \cdot \mathbf{S}_{i+k} .
\end{aligned}
$$

Note that all phonon dynamics disappeared from the Hamiltonian $H_{\text {eff }}$, but the effect of the spin-phonon interaction enters through both, the static dimerization parameter $\Delta_{\pi}$ and the different long range spin interactions.

For the local coupling the corresponding interaction strengths are

$$
\begin{aligned}
\delta^{\mathrm{loc}}= & -\frac{\Delta_{\pi}}{J}\left[(1-f)+\frac{f^{2} g^{2} Y}{2}\left(1-\frac{f}{3}\right)\right], \\
J_{0}^{\mathrm{loc}}=N\left[\frac{\omega_{0}}{J}\left\langle b_{i}^{+} b_{i}\right\rangle+\frac{1}{4 \lambda}\left(\frac{\Delta_{\pi}}{J}\right)^{2}\right. & \left.-\frac{3}{8} \lambda f\left(1-\frac{f}{2}\right)-\frac{\lambda g^{2} Y f^{3}}{16}\left(1-\frac{f}{4}\right)\right], \\
J_{1}^{\text {loc }}= & +\lambda f\left(1-\frac{f}{2}\right)+\frac{f^{2} g^{2} Y(1-\alpha)}{2} \\
& +\frac{\lambda g^{2} Y f^{3}}{2}\left(1-\frac{f}{4}\right)+\frac{f^{4} g^{4} Y^{2}}{96}(28-37 \alpha), \\
J_{2}^{\text {loc }}= & \alpha-\frac{f^{2} g^{2} Y(1-2 \alpha)}{2} \\
& -\frac{\lambda g^{2} Y f^{3}}{4}\left(1-\frac{f}{4}\right)-f^{4} g^{4} Y^{2} \frac{37}{96}(1-2 \alpha), \\
J_{3}^{\text {loc }}= & -\frac{f^{2} g^{2} Y \alpha}{2}+\frac{f^{4} g^{4} Y^{2}}{96}(9-46 \alpha), \\
J_{4}^{\text {loc }}= & \frac{9 f^{4} g^{4} Y^{2} \alpha}{96},
\end{aligned}
$$

while for the difference coupling we find

$$
\begin{aligned}
\delta^{\text {diff }}= & 2 \delta^{\text {loc }} \\
J_{0}^{\text {diff }}= & N\left[\frac{\omega_{0}}{J}\left\langle b_{i}^{+} b_{i}\right\rangle+\frac{1}{4 \lambda}\left(\frac{\Delta_{\pi}}{J}\right)^{2}\right. \\
& \left.-\frac{3}{4} \lambda f\left(1-\frac{f}{2}\right)-\frac{3 \lambda g^{2} Y f^{3}}{16}\left(1-\frac{f}{4}\right)\right],
\end{aligned}
$$

$$
\begin{aligned}
J_{1}^{\text {diff }}= & +2 \lambda f\left(1-\frac{f}{2}\right)+\frac{3 f^{2} g^{2} Y(1-\alpha)}{2} \\
& +\frac{3 \lambda g^{2} Y f^{3}}{2}\left(1-\frac{f}{4}\right)+\frac{f^{4} g^{4} Y^{2}}{24}(59-75 \alpha), \\
J_{2}^{\text {diff }}= & \alpha+\lambda f\left(1-\frac{f}{2}\right)-\frac{f^{2} g^{2} Y(3-5 \alpha)}{2} \\
& -\frac{\lambda g^{2} Y f^{3}}{12}\left(1-\frac{f}{4}\right)-\frac{f^{4} g^{4} Y^{2}}{24}(75-124 \alpha), \\
J_{3}^{\text {diff }}= & -f^{2} g^{2} Y \alpha-\frac{5 \lambda g^{2} Y f^{3}}{6}\left(1-\frac{f}{4}\right) \\
& +\frac{f^{4} g^{4} Y^{2}}{48}(32-119 \alpha), \\
J_{4}^{\text {diff }}= & \frac{21 f^{4} g^{4} Y^{2} \alpha}{48} .
\end{aligned}
$$

To point out the relevant model parameters we introduced the dimensionless coupling constants $\lambda=\bar{g}^{2} /\left(J \omega_{0}\right)$ (cf. Refs. [3,4]) and $g=\bar{g} / \omega_{0}$. Besides we used

$$
Y:=\left\langle\left(b_{i}^{+}-b_{i}\right)^{2}\right\rangle=\left\{\begin{array}{ll}
-1 & \text { if } T=0 \\
-\operatorname{coth}\left(\frac{\omega_{0}}{2 T}\right) & \text { if } T>0
\end{array},\right.
$$

as a shorthand notation. To compare our result with that of Uhrig [13], in $H_{\text {eff }}^{\text {diff }}$ we have to set $f=1$, which corresponds to the anti-adiabatic regime, and $\Delta_{\pi}=0$. Indeed we recover (except for a prefactor $1 / 2$ which in Ref. [13] enters erroneously going from eq. (11c) to eq. (13), and which consequently is also wrong in Ref. [9, eq. (3-4)]) all second order terms derived with the flow-equation method, supplemented by some new fourth order contributions.

However, in contrast to Uhrig [13], who proposed to take a thermal average within the phonon subsystem, we believe, that averaging over the phonon vacuum, i.e. setting $T=0$, is a more natural choice, especially if we are dealing with phonon-energies in the anti-adiabatic regime, where the interesting energy- and temperaturescales are small in respect of $\omega_{0}$. Therefore in the following we use $Y=-1$ and $\left\langle b_{i}^{+} b_{i}\right\rangle=0$ exclusively.

\section{TRANSITION TO A GAPPED PHASE}

A prominent feature associated with the SP instability is of course the existence of an energy gap between the ground state and lowest excitations. Considering, in a first step, the pure spin model $H_{\mathrm{s}}$, it is known that the spectrum is gapless for the Heisenberg chain with $\alpha=0$, where the lowest spinon excitations (triplet and singlet) are degenerate with the ground state at momenta $q=0$ and $\pi$ [18-20]. In contrast the system has a two-fold degenerate ground state and a gap to lowest triplet excitations at $\alpha=0.5$, the Majumdar-Ghosh point [21]. At some intermediate frustration $\alpha_{c}$ the model undergoes a transition from the gapless to the gapped phase, which is of Kosterlitz-Thouless type [22-24]. Using arguments of conformal field theory one can show that the lowest 
singlet and triplet excitations of a finite system of size $N$ become degenerate at $\alpha_{c}(N)$, where the dependence on $N$ is only weak and $\alpha_{c}(N)-\alpha_{c}(\infty) \sim N^{-2}$ [25-27]. This was used in Refs. $[28,29]$ to determine $\alpha_{c}=0.2411$.

Looking at our effective spin models $H_{\text {eff }}$ we find that the interaction with the optical phonons induces the same kind of frustrating next-nearest neighbour interaction. Therefore, without any explicit frustration $\alpha$, the effective frustration $\alpha_{\text {eff }}:=J_{2} / J_{1}$ due to the phonons can lead to a gap in the energy spectrum and to spontaneous dimerization, as was already discussed in Ref. [12]. This effect is most important in the anti-adiabatic frequency range.

Another mechanism producing a gap is (static) dimerization, i.e. an alternation of the nearest neighbour exchange integral. Taking the adiabatic limit of our effective model, $f \rightarrow 0$ and $\delta \rightarrow \Delta_{\pi}$, the ground-state energy of $H_{\mathrm{s}}+\delta \sum_{i}(-1)^{i} \mathbf{S}_{i} \cdot \mathbf{S}_{i+1}$ is known to deviate from its value at $\delta=0$ like $\delta^{4 / 3}$ [4], while the elastic energy increases with $\delta^{2}$. Therefore for all couplings $\lambda$ the groundstate energy of $H_{\text {eff }}(f=0)$ is minimal, if $\delta$ is finite. At the same time proportional to $\delta^{2 / 3}$ a gap opens in the spectrum.

By taking into account both mechanisms we can now determine the transition from the gapless phase to the gapped one. As the SP-system behaves different for the two couplings, we treat them separately, starting with the local coupling case.

\section{A. Local coupling}

In a first step we set $\Delta_{\pi}=0$ and use the level-crossing criterium $[28,29,9]$ to calculate the critical line in the $\alpha-g$ plane for different phonon frequencies $\omega_{0}$ and system sizes $N$. Since $H_{\text {eff }}^{\text {loc }}$ contains longer ranged interactions such as $\mathbf{S}_{i} \cdot \mathbf{S}_{i+3}$ and $\mathbf{S}_{i} \cdot \mathbf{S}_{i+4}$, this line slightly deviates from the line $\alpha_{\text {eff }}=\alpha_{c}$, and we have to calculate it separately. Applying the Lanczos algorithm to the effective model we obtain the critical line with high accuracy on local workstations $(N \leq 20)$. On the other hand we determine the level-crossing in the original model (1) by using the methods described in Ref. [6]. In the case $\omega_{0} / J=0.1$, the latter is complicated for the small spin phonon systems we can handle with our Lanczos diagonalization code on present day parallel computers, since the finite size gap to the first triplet excitation is a few times larger than $\omega_{0}$. If $g$ is small enough, each eigenvalues of the spin system is accompanied by a ladder of phonon states. Therefore several 'copies' of the singlet ground state lie within the singlet-triplet gap if $\omega_{0} \ll J$, and the singlet we consider for the level-crossing is not easy to determine unless we go to very large systems (see Ref. [9, Tab.II]).

Figure 2(a) and 2(b) show the critical lines in the effective (bold solid) and the original model (bold dashed) as well as the lines $\alpha_{\text {eff }}=\alpha_{c}$ (thin solid). As in the case of the pure spin model, the critical lines depend only weakly
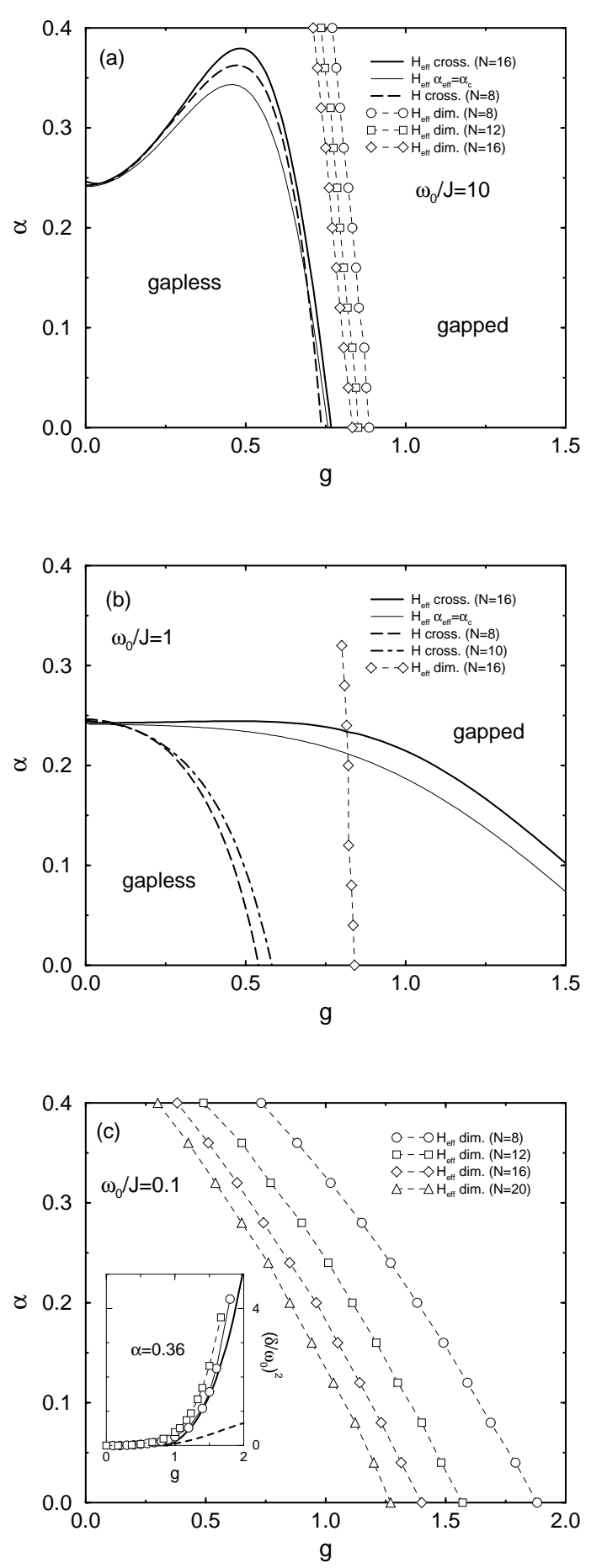

FIG. 2.: Singlet-triplet level-crossing (solid lines) and onset of dimerization (dashed with symbols) in the effective model in comparison to the level-crossing in the original model (bold dashed lines) at $\omega_{0} / J=10,1$ and 0.1 .

on $N$. We can therefore compare exact data for the orig- 
inal model and $N=8$ with data for the effective model and $N=16$. While the results differ noticeable for intermediate phonon frequency $\omega_{0} \sim 1$, the agreement is excellent in the anti-adiabatic frequency range $\omega_{0} \gg J$. With increasing $\omega_{0}$ the critical curve exhibits a remarkable upturn before crossing the abscissa, i.e. the frustration is suppressed for small spin phonon coupling, but overcritical for strong coupling. It is this feature which makes it necessary to expand (14) up to fourth order to approximate $H^{\text {loc }}$ in a correct way. A second order theory is not capable to describe the observed critical line.

Another point we can study within our effective model is the behaviour of the critical spin phonon coupling $g_{c}$ at $\alpha=0$ in the limit $\omega_{0} / J \rightarrow \infty$, i.e. the limit of the crossing point of the critical line and the abscissa. As the effects of the longer ranged interactions are rather small, we can solve the equation $\alpha_{\text {eff }}^{\text {loc }}=\alpha_{c}$. Setting $f=1$ (compare Fig. 1) and $\alpha=0$ we find

$$
\begin{aligned}
g_{c}^{2} & =\frac{P}{2 Q}+\sqrt{\left(\frac{P}{2 Q}\right)^{2}+\frac{\alpha_{c}}{Q}} \text { with } \\
P & =\frac{\omega_{0}}{J} \frac{\alpha_{c}}{2}-\frac{1}{2}\left(1+\alpha_{c}\right), \\
Q & =\frac{\omega_{0}}{J} \frac{3}{8}\left(\frac{1}{2}+\alpha_{c}\right)-\left(\frac{37}{96}+\frac{7}{24} \alpha_{c}\right),
\end{aligned}
$$

and in the limit of infinite phonon frequency $g_{c}$ approaches a finite value,

$$
\lim _{\omega_{0} / J \rightarrow \infty} g_{c}=\sqrt{\frac{8 \alpha_{c}}{3\left(1+2 \alpha_{c}\right)}} \approx 0.66,
$$

for the model with local coupling.

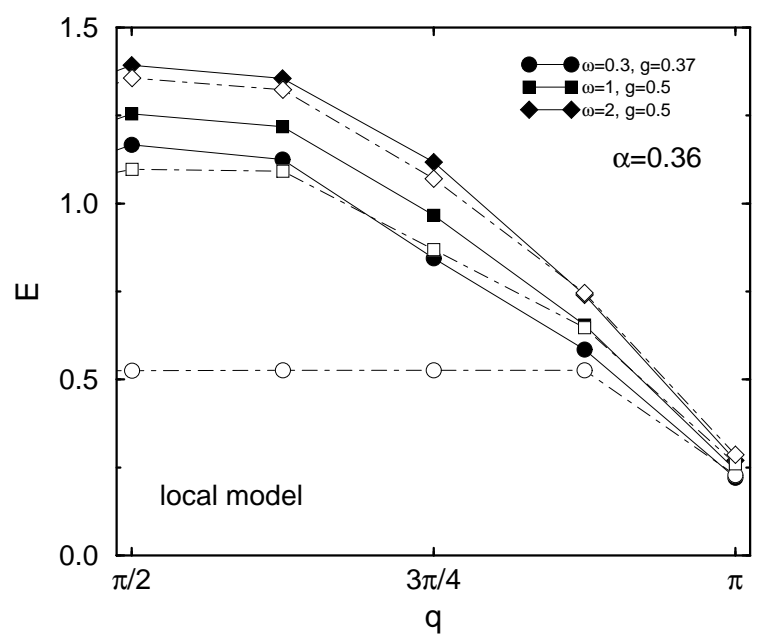

FIG. 3.: Low-lying excitations in the effective (filled symbols) and the original model (open symbols) for different frequency and local coupling $g$.
In the case small phonon frequency, $\omega_{0} \ll J$, the second transformation $\exp \left(S_{2}\right)$ looses their importance, and the effective frustration due to the spin phonon interaction is replaced by the dimerization as the relevant mechanism leading to an energy gap. We account for this effect by allowing for a finite $\Delta_{\pi}$ in our approximation. Using the Hellmann-Feynman theorem and numerical diagonalization of finite spin systems we determine $\Delta_{\pi}$ such that the ground-state energy of $H_{\mathrm{eff}}^{\text {loc }}$ is minimal. Depending on coupling strength $g$ and frustration $\alpha$ the system prefers to remain in the undimerized, gapless phase $\left(\Delta_{\pi}=0\right)$ or to develop a non-zero dimerization leading to a gap. In Figs. 2(a) - (c) we plotted these transition lines (dashed line with symbols) in addition to those obtained by level-crossing. As we already found in our study of the Holstein model of spinless fermions [16], for small $\omega_{0}$ the transition to the dimerized phase depends noticeable on the system size $N$ (see Fig. 2(c)), while the finite-size dependence is weak in the anti-adiabatic regime (cf. Fig. 2(a)). In addition, for $\omega_{0} / J=10$ the transition is consistent with the critical line determined via level-crossing.

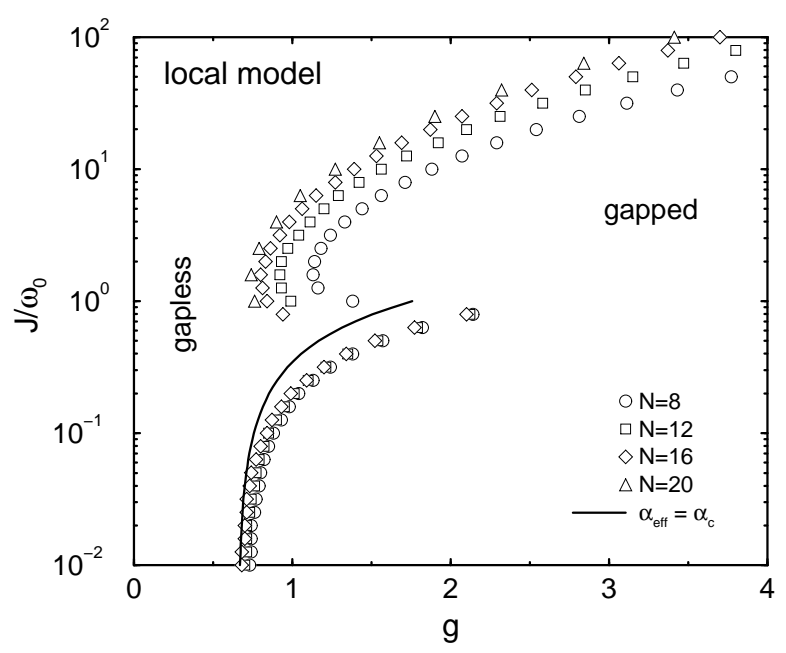

FIG. 4.: Critical coupling $g_{c}$ versus frequency for $H_{\mathrm{eff}}^{\text {loc }}$ with $\alpha=0$.

To compare properties of the original and the effective model also in the case of small phonon frequency, we consider the dimerization. As a quantity which corresponds to $\delta^{\text {loc/diff }}$ we take the static (lattice) structure factor $[6,7]$,

$$
\delta^{2}=\frac{\bar{g}^{2}}{N^{2}} \sum_{j, k}\left\langle u_{j} u_{k}\right\rangle e^{\mathrm{i} \pi(j-k)},
$$

calculated in the ground state of $(1)$, where $u_{j}=b_{j}^{+}+$ $b_{j}$. The inset of Fig. 2(c) displays $\left(\delta / \omega_{0}\right)^{2}$ for $\alpha=0.36$, $\omega_{0} / J=0.1$ (solid line), 0.316 (dashed line), and $N=8$. 
The results for the effective model (bold lines) and the original model (thin lines with symbols) agree rather well, especially for $\omega_{0} / J=0.1$.

Another feature we can compare is the dispersion of low-lying excitations. Figure 3 shows the energy of the lowest triplet excitations, calculated exactly and within our approximation. Clearly for $\omega_{0} / J=0.3$ the correct dispersion is flattened at momenta near $q=\pi / 2$. This results from the energy of the dispersionless phonons, which is added to the lowest triplet at $q=\pi$. Of course our effective model does not contain these low-lying phonon excitations. However, as soon as $\omega_{0} \gtrsim J$ the lowest excitations are due to renormalized spin interactions and well approximated by the effective model.

To collect the results of this subsection we show in Fig. 4 the critical coupling $g_{c}(\alpha=0)$ over a wide range of phonon frequencies, using both criteria for the phase transition. Symbols stand for the onset of dimerization, while the bold line corresponds to $\alpha_{\text {eff }}=\alpha_{c}$. As expected, we find that our approximation is somewhat unreliable for intermediate phonon frequency. The singularity of $g_{c}$ at $\omega_{0} / J=1$ is a manifestation of this deficiency. The correct critical line will connect adiabatic and antiadiabatic behaviour in a continuous way (compare also next subsection and [9]).

\section{B. Difference coupling}

The procedure to determine the phase transition in the SP-system with difference coupling is the same as described before. In the anti-adiabatic regime we set $\Delta_{\pi}=0$ and calculate the position of the crossing of the first triplet and the first singlet excitation for both, the original and the effective model. The results for $\omega_{0} / J=10$ and 1 are shown in Figs. 5(a) and 5(b) respectively. In contrast to the local coupling the structure of the critical line for high phonon frequency $\left(\omega_{0} / J=10\right)$ is much simpler. It appears that one would get the same shape also for a second order theory. However, to enlarge the application area of our approximation taking into account higher order contributions is still appropriate. As before, the agreement between the original and the effective model is excellent in the anti-adiabatic regime, while the deviations increase with approaching intermediate frequencies.

Calculating the behaviour of the critical coupling, $g_{c}(\alpha=0)$ in the limit of infinite phonon frequency, $\omega_{0} / J \rightarrow \infty$, we now find

$$
\begin{aligned}
g_{c}^{2} & =-\frac{P}{2 Q}+\sqrt{\left(\frac{P}{2 Q}\right)^{2}+\frac{\alpha_{c}}{Q}} \text { with } \\
P & =\frac{\omega_{0}}{J}\left(\frac{1}{2}-\alpha_{c}\right)+\frac{3}{2}\left(1+\alpha_{c}\right), \\
Q & =\frac{\omega_{0}}{J}\left(\frac{1}{16}+\frac{9}{8} \alpha_{c}\right)-\left(\frac{25}{8}+\frac{59}{24} \alpha_{c}\right),
\end{aligned}
$$

and, different to the local coupling case, $g_{c}$ tends to zero,
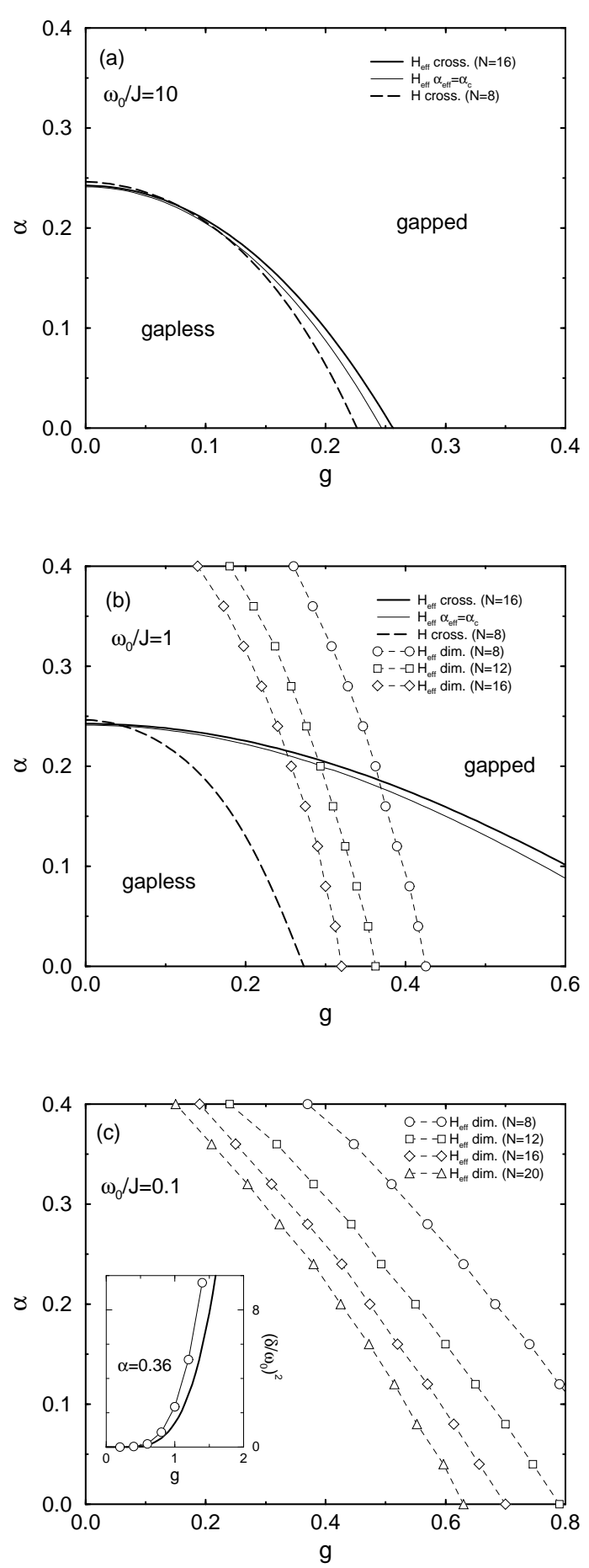

FIG. 5.: Level-crossing (solid lines) and onset of dimerization (dashed with symbols) in the effective model in comparison to the level-crossing in the original model (bold dashed lines) at $\omega_{0} / J=10,1$ and 0.1 .

$$
\lim _{\omega_{0} / J \rightarrow \infty} g_{c}=0
$$


While the $q=0$ and the $q=\pi$ phonon mode compete in the case of local spin phonon coupling, allowing for a stable gapless phase up to a critical $g$, there is no interaction with the $q=0$ mode in $H_{\mathrm{sp}}^{\text {diff }}$. Therefore the $q=\pi$ mode induces long ranged exchange more efficiently, leading to a vanishing $g_{c}$ for $\omega_{0} / J \rightarrow \infty$.

For small phonon frequencies $\omega_{0} \ll J$ again we determine the optimal dimerization $\Delta_{\pi}$ and the critical line beyond which $\Delta_{\pi}$ starts to be non-zero. The results are shown in Figs. 5(b) and 5(c). For large phonon frequency we find an unstable behaviour of $\Delta_{\pi}$, it is finite for some $g$, but vanishes before growing to substantial values again. We therefore make no attempt to fix the onset of $\Delta_{\pi} \neq 0$ for $\omega_{0} / J \gtrsim 1$.

As in the case of local coupling the dimerization in the original and the effective model agrees well for $\omega_{0} / J=$ 0.1 (inset Fig. 5(c)).

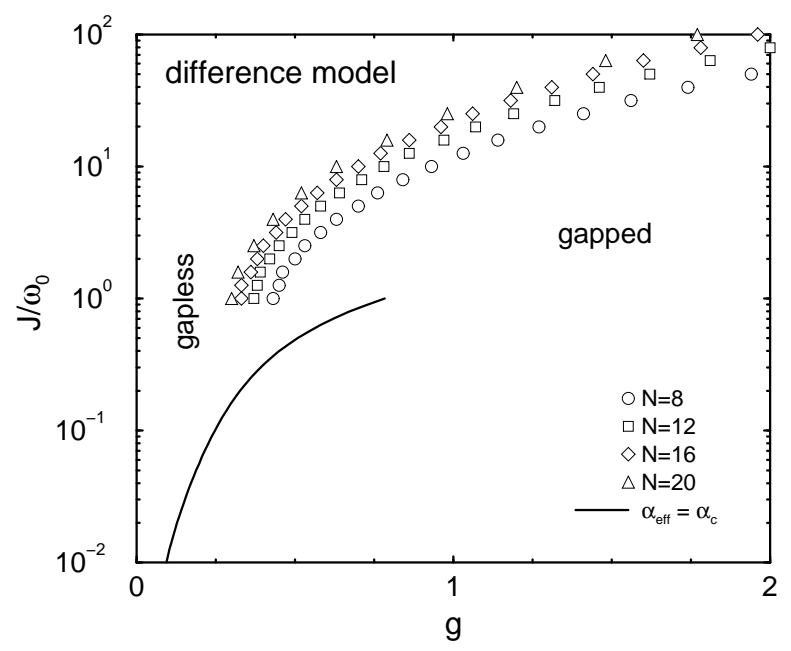

FIG. 6.: Critical coupling $g_{c}$ versus frequency for $H_{\text {eff }}^{\text {diff }}$ with $\alpha=0$.

Finally in Fig. 6 we combine the above results to obtain the phase diagram in the $J / \omega_{0}-g$ plane. Except for $\omega_{0} / J \rightarrow \infty$, where $g_{c} \rightarrow 0$, the behaviour is similar to the case of local coupling. Again at $\omega_{0} / J \sim 1$ the effective model gives a singularity in $g_{c}$, while the correct result should be continuous. Comparing the transition line with the very recent DMRG data of Bursill et al. [9], we find very good agreement in the anti-adiabatic regime (the phase transition does not change, going from the second to the fourth order effective theory). For small phonon frequency $\omega_{0} / J \rightarrow 0$ the results of Bursill et al. suggest a finite limit for $g_{c} / \omega_{0}$, while our model gives an increasing ratio of $g_{c} / \omega_{0}$. However, as the finite size effects are large for $\omega_{0} \ll J$, determining the correct value of $g_{c}$ is a delicate procedure and the exact $g_{c}$ might be much smaller than depicted in Fig. 6.

\section{GROUND-STATE PHONON DISTRIBUTION}

In the course of exact diagonalizations of the phonon dynamical model (1) we observed another interesting feature distinguishing the two mechanisms of spin phonon interaction. Turning our attention to the phonon distribution in the ground state of (1), we find

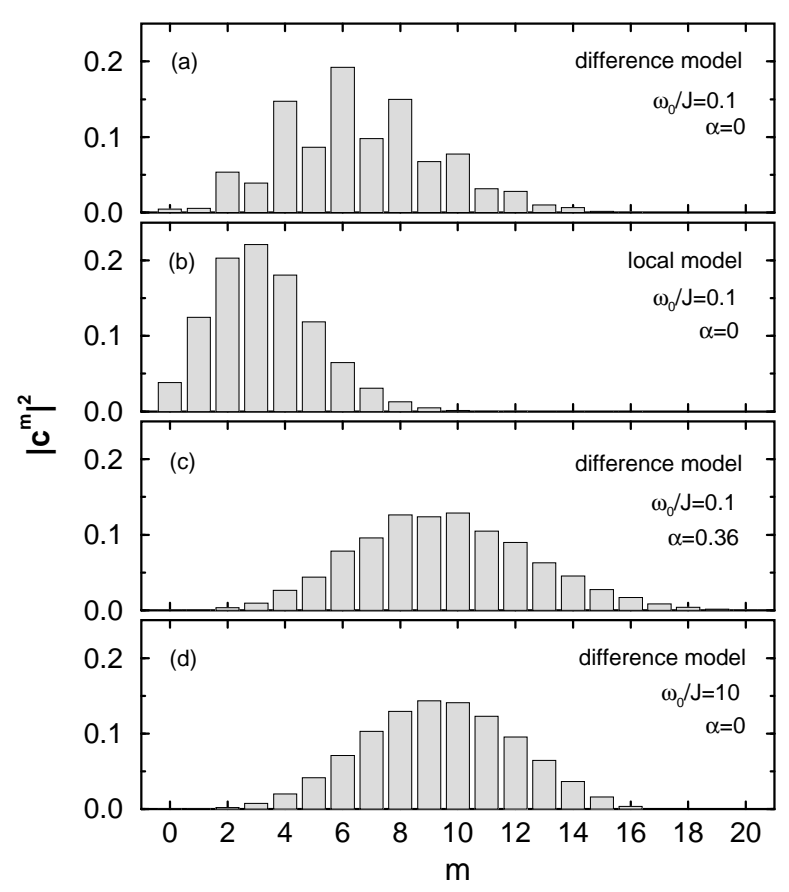

FIG. 7.: Even/odd-phonon distribution in the ground state of the model with difference coupling $(g=1.4$, $\left.\omega_{0} / J=0.1\right)$ and mechanisms that suppress the effect: local coupling, frustration and anti-adiabaticity.

for the model with difference coupling in the adiabatic regime, that the system prefers states with even phonon occupation numbers (cf. Fig. 7(a)). This behaviour reminds of a simple two-level system, more precisely the Rabi (pseudo-Jahn-Teller) Hamiltonian [30] $H=\Delta \sigma_{z}+\bar{g}\left(b^{+}+b\right) \sigma_{x}+\omega_{0} b^{+} b \sigma_{0}$, where an interaction with a bosonic degree of freedom connects the levels. Solving this model in the adiabatic strong-coupling case, $\omega_{0} / \Delta \ll 1$ and $g=\bar{g} / \omega_{0}>1$, we obtain a similar even/odd oscillation in the ground-state phonon distribution. One can understand this effect within standard perturbation theory. Starting with the two levels $\pm \Delta$ and the corresponding eigenstates $\left(\begin{array}{l}1 \\ 0\end{array}\right)$ and $\left(\begin{array}{l}0 \\ 1\end{array}\right)$, it is obvious, that adding only one phonon requires an extra amount of $2 \Delta$ exchange energy, because the system is excited to the upper level. Therefore the system prefers an even number of phonons for the ground-state wave function. In the anti-adiabatic case, $\omega_{0} \gg \Delta$, to a good approximation we can omit the $\Delta \sigma_{z}$ term and solve the model by 
unitary transformation, leading to a Poisson distribution of the phonons (coherent state).

In view of the SP system with difference coupling, the $q=\pi$ phonon is the most relevant (in fact in the ground state almost all phonons occupy this mode), and adding one phonon changes the momentum of the spin system by $\pi$. Consequently the gap between the (singlet) ground state at $q=0(N=4 k, k \in \mathbb{N})$ or $\pi(N=4 k+2)$ and the lowest singlet state at $q=\pi$, or 0 respectively, plays the role of $2 \Delta$ in the two-level system. As this singlet-singlet gap is only due to the finite size of the considered spin chain, we believe that the even/odd oscillations will disappear in the thermodynamic limit. The reduction (disappearance) of the even/odd imbalance resulting from a finite frustration with a much smaller singlet-singlet gap (zero at $\alpha=0.5$ ), cf. Fig. 7(c), can be taken as a first indication. Of course we observe a smooth phonon distribution in the anti-adiabatic case (Fig. $7(\mathrm{~d})$ ).

The model with local spin phonon coupling exhibits the usual Poisson distribution for all frequencies, since the interaction is due to the $q=\pi$ and the $q=0$ phonon mode (Fig. 7(b)).

\section{CONCLUSION}

In summary, we have studied the spin-Peierls instability of a frustrated Heisenberg spin chain coupled to optical phonons of energy $\omega_{0}$. Using the concept of unitary transformations we derive effective spin Hamiltonians, which cover the spin phonon interaction by two mechanisms, static dimerization $\Delta_{\pi}$ and long ranged exchange couplings. Both can lead to an energy gap between the ground state and lowest excitations, which is related to a Peierls instability of the spin system. In the anti-adiabatic phonon frequency range, $\omega_{0} \gg J$, we verify and extend the Hamiltonian obtained recently with the flow-equation method [13], while we recover the usual static SP model in the limit $\omega_{0} / J \rightarrow 0$.

To determine the transition to the gapped phase in the case of large phonon frequency we use the levelcrossing criterium, which proved to be very accurate for similar models $[28,29,9]$. For the two types of spin phonon coupling (local: $u_{i} \mathbf{S}_{i} \cdot \mathbf{S}_{i+1}$, and difference: $\left(u_{i}-\right.$ $\left.\left.u_{i+1}\right) \mathbf{S}_{i} \cdot \mathbf{S}_{i+1}\right)$ we consider here, the results of our effective models agree very well with data from exact diagonalization of the original, phonon dynamical model and with recent DMRG data [9] (difference coupling only). In the case of local coupling two phonon modes $(q=0$ and $\pi$ ) compete and allow for a gapless phase to exist in a wider parameter range. Furthermore we observe a non-monotonous behaviour of the phase transition line as a function of spin-phonon interaction and frustration $\alpha$. With difference coupling spins and phonons interact almost only through the $\pi$-mode, which is able to dimerize the system most efficiently.

For phonon frequencies $\omega_{0} \lesssim J$, we determine the phase transition by means of the static dimerization $\Delta_{\pi}$, which changes from zero at small spin phonon coupling to a finite value beyond a critical coupling.

At intermediate frequency the situation remains unsatisfactory as the critical coupling behaves discontinuously. Here numerical methods, including the full phonon dynamics, still provide the only reliable tool to study the transition. Nevertheless the proposed effective models help to understand the physical mechanisms leading to spontaneous dimerization of the interacting spin-phonon system.

\section{ACKNOWLEDGEMENT}

We thank J. Schliemann for valuable discussions. Some computations were done at LRZ München, HLRZ Jülich, HLRS Stuttgart, and GMD Bonn. H.F. acknowledges financial support from the Graduiertenkolleg 'Nichtlineare Spektroskopie und Dynamik' at the University of Bayreuth.

[1] For a review see J.S. Miller (ed.), Extended Linear Chain Compounds, Vol. 3, Plenum, New York 1983, Chapter 7.

[2] M. Hase, I. Terasaki, K. Uchinokura, Phys. Rev. Lett. 70, 3651 (1993).

[3] E. Pytte, Phys. Rev. B 10, 4637 (1974).

[4] M.C. Cross, D. Fisher, Phys. Rev. B 19, 402 (1979).

[5] C. Gros, R. Werner, Phys. Rev. B 58, R14677 (1998).

[6] G. Wellein, H. Fehske, and A.P. Kampf, Phys. Rev. Lett. 81, 3956 (1998).

[7] B. Büchner, H. Fehske, A.P. Kampf, and G. Wellein, Physica B, accepted (1998).

[8] L.G. Caron, S. Moukouri, Phys. Rev. Lett. 76, 4050 (1996).

[9] R.J. Bursill, R.H. McKenzie, C.J. Hamer, condmat/9812409.

[10] A.W. Sandvik, R.R.P. Singh, D.K. Campbell, Phys. Rev. B 56, 14510 (1997); J.E. Hirsch, E. Fradkin, Phys. Rev. B 27, 1680 and 4302 (1983); R.H. McKenzie, C.J. Hamer, D.W. Murray, Phys. Rev. B 53, 9676 (1996).

[11] H. Zheng, Phys. Rev. B 56, 14414 (1997).

[12] K. Kuboki, H. Fukuyama, J. Phys. Soc. Jap. 56, 3126 (1987).

[13] G.S. Uhrig, Phys. Rev. B 57, R14004 (1998).

[14] F. Wegner, Ann. Phys. 8. Folge, Bd. 3, 77 (1994).

[15] W. Geertsma, D. Khomskii, Phys. Rev. B 54, 3011 (1996); D. Khomskii, W. Geertsma, M. Mostovoy, Czech. Journ. Phys. 46 Suppl. S6, 32 (1996); condmat/9609244.

[16] A. Weiße and H. Fehske, Phys. Rev B 58, 13526 (1998).

[17] J.R. Schrieffer, P.A. Wolff, Phys. Rev. 149, 491 (1966).

[18] J. des Cloizeaux, J.J. Pearson, Phys. Rev. 128, 2131 (1962). 
[19] T. Yamada, Prog. Theor. Phys. Jpn. 41, 880 (1969).

[20] L.D. Faddeev, L.A. Takhtajan, Phys. Lett. A 85, 375 (1981).

[21] C.K. Majumdar, D.K. Ghosh, J. Math. Phys. 10,1388 , 1899 (1969).

[22] J.L. Black, V.J. Emery, Phys. Rev. B 23, 429 (1981).

[23] M.P.M. den Nijs, Phys. Rev. B 23, 6111 (1981).

[24] F.D.M. Haldane, Phys. Rev. B 25, 4925 (1982)

[25] J.L. Cardy, J. Phys. A 19, L1093 (1986); ibid. 20, 5039 (1987).

[26] V.J. Emery, C. Noguera, Phys. Rev. Lett. 60, 631 (1988).

[27] I. Affleck, D. Gepner, H.J. Schulz, T. Ziman, J. Phys. A 22, 511 (1989).

[28] K. Okamoto, K. Nomura, Phys. Lett. A 169, 433 (1992).

[29] G. Castilla, S. Chakravarty, V.J. Emery, Phys. Rev. Lett. 75, 1823 (1995).

[30] Yu.E. Perlin, M. Wagner (eds.), The dynamical JahnTeller effect in localized systems, MPCMS Vol. 7, NorthHolland, Amsterdam 1984, and references therein. 